\title{
Social Work with Veterans in Rural Communities: Perceptions of Stigma as a Barrier to Accessing Mental Health Care
}

\author{
Rebecca L. Stotzer \\ Julia M. Whealin \\ Dawna Darden
}

\begin{abstract}
The nearly decade long efforts in the Global War on Terror have led to increasing numbers of Veterans of the armed services returning to rural locations, but little is known about their needs. However, recent research suggests that rural Veterans face a host of issues, but perhaps more importantly, are facing heightened levels of stigma in rural areas related to their health and mental health. This paper examines how mental health stigma in the military may feed into stigma in rural communities and serve as an additional barrier for Veterans in rural areas who are struggling with mental health concerns. Recommendations for the unique role of social workers in serving these Veterans, as well as addressing community issues around stigma, are addressed.
\end{abstract}

Keywords: Mental health stigma; Veterans; OIF/OEF; rural social work; barriers to care

\section{INTRODUCTION}

The Global War on Terror's nearly decade of conflict in the Middle East has resulted in an increased number of Veterans returning from deployments with a variety of mental health issues. However, help for those issues is not evenly distributed across Veterans. Over $40 \%$ of the Veterans of the wars in Iraq and Afghanistan are from rural areas, and existing research shows that rural Veterans Health Administration (VA) patients report poorer mental health status and reduced access to care compared to VA patients in urban or suburban areas (e.g., Weeks, Kazis, Shen, Cong, Ren, Miller, et al., 2004; West \& Weeks, 2006). Obviously, logistical factors, including longer waiting periods, limited choice of health professionals, and poorer public transportation, limit access to and use of services in rural settings (Aisbett, Boyd, Francis, Newnham, \& Newnham, 2007). However, efforts by the VA to overcome logistical barriers by expanding services to rural areas have not led to the expected increase in service utilization (Fisher, 2003). Identifying logistical barriers answers questions about whether or not a Veteran can access care, but offers little insight into whether or not a Veteran will access care.

This information is particularly troubling given the research that demonstrates the high level of need among rural Veterans. Research has suggested that Veterans located in rural settings generally have worse health-related quality of life than Veterans living in urban locations (Weeks, et al., 2004). Although in greater need of mental health care services, rural Veterans face many challenges to receiving care that are different from

\footnotetext{
Rebecca L. Stotzer, Ph.D., is an associate professor at the Myron B. Thompson School of Social Work, University of Hawai i, Mānoa. Julia M. Whealin, Ph.D., is a Clinical Psychologist at the National Center for Posttraumatic Stress Disorder, Pacific Islands Division, VA Pacific Island Health Care System, Honolulu and an adjunct associate professor at the Department of Psychiatry, John A. Burns School of Medicine, University of Hawaii, Honolulu. Dawna Darden, MSW, is a doctoral student at the Myron B. Thompson School of Social Work, University of Hawaii, Honolulu and has an appointment at the National Center for Posttraumatic Stress Disorder, Pacific Islands Division, VA Pacific Island Health Care System, Honolulu.
} 
urban Veterans. Few studies have looked beyond the logistics of care delivery in rural settings to understand the reasons why rural Veterans may or may not choose to utilize existing services. In non-Veteran populations, rural people have been identified to have three main factors that can act to prevent them from accessing care: sociodemographic factors (e.g. gender, age, and marital status), illness-related factors (e.g. comorbidity, psychological distress), and attitudinal factors (e.g. stigma, stoicism, self-efficacy; Jackson, Judd, Komiti, Fraser, Murray, Robins, Pattison, \& Wearing, 2007). Of these three factors, attitudinal factors are the least understood, but research suggests that the more stigma a rural-dwelling person perceives, the more their reported negative attitudes toward help-seeking (Wrigley, Jackson, Judd, \& Komiti, 2005).

Research focusing on rural communities and their attitudes toward mental health and mental health services, as well as existing research on military culture and attitudes toward mental health and mental health services, suggests that rural Veterans may face significant social challenges in attaining services in their communities. Both sets of cultural values, rural and military, emphasize stoicism, self-reliance, and negative attitudes toward help seeking, as well as issues related to privacy concerns, confidentiality, and heightened perceptions of community-based stigma. Given social work's unique place and expanded role in rural environments, particularly in community mental health (e.g., Jerrell \& Knight, 1985; Landsman, 2002; Riebschleger, 2007), rural social workers can serve as a key point of outreach, advocacy, and treatment for rural Veterans. However, there is little social-worker specific information currently available to help guide providers in their attempts to serve active military, national guard, reserve, and Veteran populations (Savitsky, Illingworth, \& DuLaney, 2009). The purpose of this paper is 1) to review existing research on mental health stigma, 2) to outline the significant challenges that rural Veterans face compared to urban counterparts, and 3) to offer suggestions for social workers working in rural areas to facilitate rural Veterans finding and receiving the benefits and treatments they earned following their service to their country.

\section{STIGMA AND MENTAL HEALTH}

Stigma concerns negative stereotypes, social status loss, and discrimination related to a particular perception of difference (Link \& Phelan, 2001). It is a multidimensional concept that brands certain classes of people as inherently "different." Goffman (1963) argued that stigma "spoils" a person's identify, and asserted that "we believe the person with a stigma is not quite human. On this assumption we exercise varieties of discrimination through which we effectively, if often unthinkingly, reduce life chances" (p. 5). Jones, Farina, Hastorf, Markus, Miller, and Scott (1984) explained that we all are "marked" in some way, whether it is simply having blue eyes, being tall, etc., but stigma is the application of a negative valuation on that mark. Thus, stigma is based on assumptions, stereotypes, and value judgments about this marked identity that are shared among a wide population as well as understood by the person bearing the marked identity.

Stigma toward those with mental health issues has been found to be pervasive and often severe (Wahl, 1999). A variety of negative stereotypes have been applied to people 
struggling with mental illness, such as the perception that mental illness leads to violence, even though research suggests that people with mental illness are not more violent than the general population (Levin, 2001). Mental illness has been called a "concealable" stigma, because in most cases it is not obvious to a stranger (Hinshaw, 2007). A person who is dealing with mental health concerns often spends a large portion of mental and emotional energy attempting to conceal their mental health concern, and attempting to judge others' opinions and perceptions of their behaviors (Smart \& Wegner, 1999). A large motivator for keeping their mental health concern concealed is to avoid a label being applied to them. The desire to maintain concealment can result in refusal to acknowledge an issue or pursue mental health treatment to avoid being affiliated with the stigmatized group (Corrigan, Watson, Byrne, \& Davis, 2005). This desire to conceal an identity that is stigmatized is pervasive, even when disclosure can result in many mental health benefits and well-being (Chaudoir \& Quinn, 2010). In a general population, greater perceived and self-stigma is related to reduced help-seeking from professional sources (Barney, Griffiths, Jorm, \& Christensen, 2006). Although most populations are aware of, and suffer the consequences of mental health stigma, military-related populations are at particular risk for experiencing the negative effects of stigma, and as a result, avoid seeking care to prevent real or imagined discrimination and/or loss in social status.

\section{Veteran and Military-related Mental Health Stigma}

Traditionally, the military services have embraced normative beliefs consistent with the masculine gender role identity. Traditional attitudes common in military groups include valuing independence, self-reliance, competition, power, strength, and emotional control. Although recent outreach and education efforts by the military and VA have begun to change this culture (e.g., Adler, Bliese, McGurk, Hoge, \& Castro, 2009; Reivich, Seligman, \& McBride, 2011), traditionally military groups tend to eschew mental health services. Instead, coping tactics viewed as suitable means for dealing with stress include behaviors such as emotion suppression and substance abuse (Lorber, \& Garcia, 2010). Not surprisingly, conformity with masculinity norms is associated with less use of health care services (Boman \& Walker, 2010).

The belief that one ought to be able to handle mental health problems on their own continues to be a commonly reported barrier to care (e.g., Sayer, Friedemann-Sanchez, Spoont, et al., 2009; Stecker, Fortney, Hamilton, et al., 2007). Beliefs held by Veteran and military groups suggest that this belief may most strongly reflect concerns about the mental health attitudes of, and potential discrimination by, others, as opposed to internalized beliefs. In one of the most comprehensive studies about Veterans and mental health stigma to date, Hoge, Castro, Messer, McGurk, Cotting, and Koffman (2004) evaluated Active Duty Soldiers and Marine units returning from Iraq or Afghanistan $(\mathrm{N}=$ $6,153)$ to identify specific barriers to mental health care. The most commonly endorsed items indicating stigma included "My unit leadership might treat me differently" $(36.5 \%)$, "I would be seen as weak" (35.4\%), "Members of my unit might have less confidence in me" $(34.2 \%)$, "It would harm my career" $(27.0 \%)$, followed by "There would be difficulty getting time off from work for treatment" $(26.3 \%)$. Their findings 
indicated that the majority of the returnees acknowledged a need for help with mental health issues (78-86\%). However, less than one third of returnees (13-27\%) who acknowledged a need for help accessed available mental health services. When asked about the barriers to obtaining treatment, the service members classified as in need of mental health care endorsed negative stigma about treatment as the greatest barriers to service utilization (Hoge, et al., 2004). Warner, Appenzeller, Mullen, Warner, and Grieger (2008) had similar findings in a study of 2,678 soldiers deploying with a brigade combat team, soldiers $(n=2,678)$ reported their concerns were that "Members of my unit might have less confidence in me" (20.8\%), "My unit leadership might treat me differently" (22.4\%), "It would harm my career" (18.5\%), and "I would be seen as weak" $(17.8 \%)$.

These perceptions of stigma from unit members and superiors have direct behavioral effects. Among previously deployed national guard soldiers who screened positive for PTSD, depression, or alcohol dependence, many reported significant discomfort reporting mental health symptoms and a belief that their superiors would not encourage mental health treatment (Kline, Falca-Dodson, Sussner, Ciccone, Chandler, Callahan, \& Losonczy, 2010). Additionally, 59\% of this group indicated that they did not report symptoms during military Post-Deployment Health Reassessments in order to avoid "medical hold." Nearly half of those screening positive for PTSD and almost $90 \%$ of those screening positive for alcohol dependence reported no treatment in the past 12 months (Kline, et al., 2010).

Recent research with OEF/OIF military populations (e.g., Wright, Cabrera, Bliese, Adler, Hoge, \& Castro, 2009) suggests that military community attitudes and internalized-stigma are two interacting factors that influence service members' willingness to get help. Thus, there is a tremendous need to better understand factors known to impact Veterans' decisions to access mental health care. Notably, Active Duty Service Members and Veterans with mental health problems, arguably those most in need of care, are up to twice as likely as those without mental health problems to report stigma as a barrier to health-care use (e.g., Hoge, et al. 2004). Pietrzak, Johnson, Goldstein, Malley and Southwick (2009) examined beliefs about the effectiveness of mental health care in a sample of 272 predominantly reservist and national guard OEF/OIF Veterans in Connecticut. The participants who screened positive for a psychiatric disorder scored higher on scales measuring perceptions of stigma and logistical barriers to care. Additionally, negative beliefs about the effectiveness of psychotherapy and psychotropic medications predicted increased stigma and barriers to care. Negative beliefs about mental health care were also associated with decreased likelihood of psychotherapy and medication visits. In a sample of Vietnam and OEF/OIF Veterans, Stecker, Fortney, Hamilton, and Ajzen (2007) found that the belief that one "ought to be able to handle mental health problems on their own" was the most commonly reported barrier to care. In a qualitative study examining the determinants of PTSD treatment initiation in Veterans (Sayer, et al., 2009), pride in self-reliance was a strong deterrent to treatment seeking.

These studies highlight the concerns of service members, and the degree to which they are attuned to perceptions of mental health stigma in their environments. In addition, the elevated levels of psychological problems reported by new OEF/OIF Veterans 
emphasize the need to reduce the stigma as members of this population separate from the military and return to civilian communities. To date, however, relatively little research has been conducted to assess the link between civilian community factors and perceptions of stigma and barriers to care. However, an emerging body of research suggests that rural communities may have a heightened level, or produce the perception of a heightened level, of mental health stigma.

\section{Rural Culture and Perceptions of Community Stigma}

Most studies evaluating rural populations do not attend to the contextual community characteristics of rural settings that likely play a role in impacting individual attitudes about and willingness to use mental health services (Boyd, Hayes, Sewell, Caldwell, Kemp, Harvie, et al. 2008; Jackson, et al., 2007). The source of stigma does not appear to be the mental health system itself, but rather the communities' beliefs and attitudes about mental health (Crawford \& Brown, 2002). Evidence suggests that rural residents incorrectly underestimate the prevalence of mental health problems in their communities (Bartlett, Travers, Cartwright, \& Smith, 2006), perhaps implicitly contributing to the stigma by demonstrating a "that doesn't happen here" attitude. Regardless of severity of mental illness symptoms, and despite similar rates of mental illness, rural residents are also less likely to access mental health services than urban residents (Dahlberg, Fosell, Damstrom-Thakker, \& Runeson, 2007). This may be due in part to the high levels of perceived stigma in rural communities, where rural residents with mental health problems still strongly identify with their rural communities and want to belong (Ekeland \& Bergem, 2006).

Although the perception of stigma has been found in the general population as a barrier to seeking services (e.g., Barney, et al., 2006), in rural settings, recent research has identified fundamental, contextual factors that result from characteristics of social networks that impact help-seeking and service use (Boyd, et al., 2008). For example, in rural settings, community members, despite being separated by many miles due to geographical constraints, can have intimate knowledge of each other's lives due to local gossiping networks (e.g., Aisbett et al., 2007; Parr \& Philo, 2003; Parr, Philo, \& Burns, 2004). This is the opposite social geographical characteristics experienced in urban settings, where community members may be physically proximate but are often socially distant from each other. As a result, contextual factors of rural settings - including a combination of being socially visible and having strong social networks - have the potential to heighten both community social stigma and internalized stigma related to use of mental health services.

Research on non-Veteran populations supports the role of contextual factors in impacting attitudes about and use of mental health services (e.g., Boyd, et al., 2006; Boyd, et al., 2008). Wrigley et al. (2005) found that the two main reasons not to seek help from a general practitioner for mental health problems were 1) embarrassment (see also Hoyt, Conger, Valde, \& Weihs, 1997) and 2) not knowing who to seek help from. At the same time, they found a relationship between perceived stigma such that those who reported higher rates of perceived stigma also demonstrated more negative attitudes toward help seeking, drawing a link between stigma and its impact on help-seeking. To 
give additional evidence for these qualitative results, another study asked rural residents to evaluate a vignette of a man exhibiting symptoms of mental illness, and $67 \%$ of rural residents said he would be discriminated against in their community (Bartlett, et al., 2006). In Hoyt et al. (1997), when "place" and both measures of stigma and loss of privacy were entered into the same regression model, the effects of place disappear, suggesting that issues of embarrassment related to confidentiality and anonymity moderate the relationship between place and help-seeking.

Qualitative studies consistently reveal that recognition as a person with a mental health issue via a community's informal social networks has a stigmatizing effect. One qualitative study of rural and urban adolescents indicated that those in rural settings identified rural gossip networks as limiting anonymity. In addition, among rural adolescents, fear of social stigma and exclusion after revealing a mental health problem was seen as a barrier to obtaining services (Quine, Bernard, Booth, Kant, Usherwood, Alperstein, et al., 2003). In another study of rural college students, participants noted that gossip networks and social visibility within rural settings play an extremely influential role in stigmatizing those who had received mental health services (Aisbett, et al., 2007). Furthermore, qualitative studies that have been done with non-Veteran populations suggest that those in rural settings are more likely to see those who use mental health services as weak and that their use of services suggests a lack of self-reliance (Boyd, Aisbett, Francis, Kelly, Newnham, \& Newnham, 2007). For example, Wrigley, Jackson, Judd, and Komiti (2005) found that more negative attitudes toward help-seeking for schizophrenia and depression were related to higher endorsements that these problems were caused by a weakness in character.

Fuller, Edwards, Proctor, and Moss (2000) found that a rural culture of self-reliance was composed of two features, first, not being allowed to be weak, and second, being suspicious of outsiders (including mental health providers from outside the community). This attitude leads to stigma and decreased probability that rural people will label their mental distress as a mental health problem. Fuller et al. (2000) suggested that even in the cases where rural residents recognize their mental distress despite the stigma, they may still not see the "system" as a source of help because of their resistance to be seen as the community "nutcase." When broken apart, attitudes about the importance of stoicism and general self-efficacy were found to be important predictors of help seeking (Judd, Jackson, Komiti, Murray, Fraser, Grieve, \& Gomez, 2006). However, the values of selfreliance are not insurmountable. For example, having sought help in the past for mental health concerns was related to more positive attitudes toward help-seeking (Jagdeo, Cox, Stein, \& Sareen, 2009), though this may not be a consistent relationship for men, who may be more prone to believe in agrarian values (Hoyt, et al., 1997). These studies highlight how rural community values and attitudes that prevent help-seeking can be assuaged by positive interactions with mental health systems.

There was evidence that in rural settings many people felt that the resources available to them were inadequate or of poor quality, hence keeping them from seeking help. Human and Wasem (1991) in an overview of rural health concerns in the United States wrote that there is often a difference between accessibility, which is whether or not a client is able to access services, vs. acceptability, which is whether or not a service is 
being offered in a manner consistent with local values and for a rural setting. Bartlett et al. (2006) in a survey in rural Queensland found that 58\% of respondents felt that available services were of poor quality. Wrigley et al. (2005) found that when inquiring about why people did not go to a general practitioner (GP) for help with mental health issues, those who felt that their GP would not be helpful with mental health related inquiries were $91 \%$ less likely to discuss mental health concerns with their GPs. The size of the community may also play a role in the perception of service availability as well as confidentiality and privacy - the smallest communities and the largest rural locations have been shown to have the lowest rates of access compared to moderately sized locations (Hoyt, et al., 1997; Judd, Jackson, Komiti, Murray \& Fraser, 2007).

In regard to rural social work in particular, the role of rural social workers is varied. Fiske (2003) identified many of the strengths (such as the strong connections made with other professionals, strengthened professional teamwork, etc.) and challenges of being a social worker in a rural area (such as being the only social worker, confidentiality concerns, etc.). Authors have argued that "as specialist services are few, rural social workers work across a range of methodologies and interventive strategies" (Green, 2003, p. 10) and that this focus on generalist models of interdependence, cooperation, and teams are in fact most consistent with rural values. In contrast with the stigma, attitudes, and beliefs held by rural persons, a study of rural social workers (Riebschleger, 2007) found that rural social workers also had concerns about stigma against themselves as mental health professionals. The author indicates rural dwellers' barriers to care can stem from the perception that mental health and social service providers (including social workers) would see them as simpletons, critique their dialects, or assume they are like the stereotypes of rural people in the media (such as hillbillies). Green (2003) argues that social workers who are clearly integrated into the community, and are seen as citizens (as opposed to "visitors" or "organizers") can effectively counteract some of these common concerns with "outsiders" looking down on rural residents who are in need of services.

\section{RURAL VETERANS AND STIGMA}

Although there is burgeoning research on rural environments and a long history of research on Veterans, few studies have focused specifically on rurally located Veterans. However, similar to findings about non-Veteran rural population, patterns of care utilization have shown that rural Veterans access care less often than urban Veterans, and that older Veterans were more reliant on VA care while younger Veterans used the private sector (Weeks, Mahar, \& Wright, 2005). Given that military culture and rural culture share many features that promote perceptions of mental health stigma and discourage mental health treatment-seeking, it seems likely that Veterans residing in rural locations may experience stigma as a barrier to care at a heightened level than the general population.

Among the few studies that addressed rural Veterans specifically, there is some indirect evidence that rural community characteristics impact Veterans as well. For example, Weeks et al. (2004) found that rural Veterans had lower overall health (looking at both physical and mental health) related quality of life compared to urban or suburban Veterans. Wallace, Weeks, Wang, Lee, and Kazis (2006) found that although Veterans in 
rural settings had less frequency of psychiatric disorder, for those who did, they bore a much higher disease burden as measured by poorer quality of life. One qualitative study which examined implementing integrated health care services in rural communities (Kirchner, Cody, Thrush, Sullivan, \& Rapp, 2004) noted that contextual factors related to the culture of the community were important in the success of clinical interventions. This highlights how not all rural communities may be contributing to heightened levels of stigma. It also suggests the need for community interventions targeting rural communities in regard to mental health education.

Studies have also found some differences in rural Veteran's experiences that highlight additional areas for intervention. For example, research (e.g., Costello, Pugh, Steadman, \& Kane, 1997; Molinari, Boeve, Kunik, \& Snow-Turek, 1999) suggest that Veteran's social networks are stronger in rural vs. urban settings. Comparisons between chronically ill, older male Veteran patients (43 from rural settings and 48 from urban areas) found that rural Veterans reported more social contact in their home communities than urban Veterans, despite the fact that the Veterans in the rural sample were more likely to be older, widowed, and living alone (Costello, et al., 1977). These results suggest that in addition to strengthening communities, social workers can help rural Veterans by creating interventions that promote strong social networks and social supports.

VA attempts at reaching rural Veterans and providing high quality care have met with significant challenges. In an examination of the Community-based Outpatient Clinics (CBOC)s, Chapko, Borowsky, Fortney, Hedeen, Hoegle, Maciejewski, and Lukas (2002) found that CBOCs rated similarly to their parent VAMCs on many factors, (e.g., perceptions of access, emotional support, care coordination, etc.), but were still rated as having lower average quality of care scores. Mohamed, Neale, and Rosenheck (2009) conducted research on the Mental Health Intensive Case Management (MHICM) program, which is national in scope, in order to identify variance in rural Veterans receiving services and service delivery. Veterans residing in rural areas had less contact with MHICM staff and they were less likely to receive recovery-oriented services such as rehabilitation services, psychotherapy, crisis intervention, screening for medical problems, housing, or vocational support. Mohamed et al. (2009) argued that although these national programs have been established by the VA, that rural Veterans face specific challenges in accessing these services, and that there is an ongoing need for intensive case management services.

\section{DISCUSSION}

Many Veterans lived in rural communities when they chose to enter the military, and many return to rural communities after they have completed their service. With the high level of stigma against seeking mental health services in rural communities and within the military, rural Veterans who are suffering from mental health disorders are at risk for discounting their problems, regardless of their severity. Social workers are in a unique position, due to their training at all levels of practice, to educate rural Veterans, families, and communities in order to help overcome stigmatizing attitudes and fears. 
Most social workers are not trained specifically to deal with rural issues, and social workers in the community are in need of resources to better understand and meet the needs of active military, national guard, reserve, and Veteran populations (Savitsky, Illingworth, \& DuLaney, 2009). In recognition of the unique needs of rural Veterans, the Office of Rural Health within the VA was created in 2006 to improve access and the overall quality of care for Veterans in rural and remote areas. ORH develops evidencebased policies and innovative practices that support the unique needs of those residing in rural areas. In the past two years, $\$ 500$ million was appropriated for new health initiatives for rural Veterans ranging from expansion of specialty services provided via telehealth to community and rural provider outreach and education. Additionally, other centers of excellence within the VA provide educational materials to providers who work with military populations. For example, the National Center for PTSD provides Clinicians' Manuals, as well as a variety of on-line courses on topics such as treatment for PTSD and understanding military culture online at http://www.ptsd.va.gov/professional/index.asp. Because up to $50 \%$ of rural locations in the United States are still without Internet access, many of these materials are available on CD upon request. Finally, this literature review suggests that there are many strategies that both civilian and non-civilian social workers

can employ to best serve the Veterans in their rural communities, and to combat both the military and rural stigmas against mental illness and mental health treatment-seeking.

\section{Prevention}

There is emerging evidence that addresses the need to prevent Veterans from suffering negative mental health as a consequence of their military service (Warner, Appenzeller, Mullen, Warner, \& Grieger, 2008). Of the soldiers who had previously deployed, $20.7 \%$ had sought MHC upon their return and $84.8 \%$ reported that they had received Army psychoeducational "Battlemind" training, now called "Resiliency Training" (Adler, Bliese, McGurk, Hoge, \& Castro, 2009). Soldiers in this brigade who received this training were 1.56 times more likely to report that they would seek treatment if screening results were positive or if they perceived a problem. Warner and colleagues (2008) conclude that efforts to reduce stigma via education prior to a problem emerging may be helping to decrease stigma and then increase treatment seeking.

\section{Culturally-appropriate Therapeutic Intervention}

Culturally-appropriate care for rural military populations involves understanding the Veteran's values and belief system. For some Veterans, this may mean respecting the role that independence, self-reliance, strength and emotional control has in a Veteran's life. At the same time, social workers should understand that once trust is established, a Veteran may be more open to broach subjects related to mental health symptoms when asked. It is helpful to openly discuss concerns a Veteran may have about stigma and confidentiality, especially in rural areas where "everyone knows everyone." When a Veteran who endorses mental health stigma is suffering from mental health problems, it is helpful to normalize both the commonality of the symptoms and the discomfort with talking about them. Finally, in therapy it may be helpful to openly discuss that, while independence, self-reliance, and emotional control can be strengths, rural and military values applied too 
rigidly to everyday civilian situations are maladaptive. For instance, research shows that willful suppression of emotions tends to lead to higher levels of disturbing thoughts and emotions, and is implicated in PTSD (Shipard \& Beck, 2005). Once Veterans feels that they have 'permission' to confidentially discuss symptoms, they will be better able to identify and address their problems (Lorber, \& Garcia, 2010).

\section{Strengthening Social Relationships and Networks}

The existing literature highlights the need for both honoring and utilizing existing social networks and supports for Veterans in rural locations. Those relationships, although at time can lead to a perception of increased "gaze" from other community members that raise concerns about treatment seeking, can also serve as significant strengths in finding mechanisms to provide care to rural Veterans. In regard to stigma specifically, Veterans repeatedly cited their concerns that admitting mental health concerns would result in lost face to peers, superiors and families, decreased sense of masculinity, and concerns about their job. Interestingly, a study examining Active Duty Soldiers three months after deployment showed that Soldiers who rated their leaders more favorably and who reported higher unit cohesion were more likely to report lower stigma scores (Wright, et al., 2009). These results highlight how critical relationships are, before separating from the military, as well as post-separation. Social workers are in a key position of being able to work with service members and their families to provide services that use strong existing relationships among Veterans that can help counteract the negative stereotypes about treatment-seeking. Community leaders (such as clergy), cultural brokers, and strong Veteran role models all serve as excellent collaborators in such endeavors. Given that social workers, particularly civilian social workers, often serve multiple roles within a health care system in rural areas, they have a significant opportunity to intervene with Veterans and families in ways that directly target family functioning, and in the long run may decrease perceptions of stigma, and overcome a barrier to care for some Veterans.

\section{Coordinating Military and Civilian Services}

As the VA recognized the difficulty in providing adequate and high quality services to rural areas, it has begun exploring the variety of mechanisms to provide services outside of simply starting entirely new facilities. The possibility of coordinating with existing community mental health services and systems has been strongly encouraged by policy groups such as the National Rural Health Association (2004). This policy organization has been advocating for policy changes that would allow the VA and other services (such as local community health centers, vet centers, and other rural providers) to cooperate in a more meaningful and coordinated fashion. Savistky et al. (2009) stated that "civilian social workers must acknowledge their responsibility to reach out to their military counterparts in an effort to achieve the most streamlined system of care possible. When military and civilian systems operate in isolation, an opportunity to coordinate well-informed and effective intervention is lost" (p. 337). In their generalist roles, rural social workers often interact with many systems and professions. In this space, rural social workers are poised to help build those connections between systems. However, this 
requires civilian social workers to become at least marginally versed in the language and systems of the military and the VA. In order to best serve Veterans, particularly those who have seen combat over the last decade, all social workers need a basic understanding of the military and VA. However, where urban social workers may more easily be able to refer urban Veterans to VA services, rural social workers need to 1) look harder for Veterans who may not be accessing because of stigma concerns, and 2) may need to have an increased level of knowledge about military and VA systems since community access to those resources and information may be limited. This may mean becoming knowledgeable about the VA's current proliferation of online services (such as My HealthEvet), the possibilities for telehealth (such as completely online individual and group therapy available via teleconferencing), and emedicine as means to overcome logistical barriers to care. The more clearly rural providers know about options for Veterans, even in their rural communities, the less stigmatizing treatment-seeking will become as it is normed in the community.

\section{Community Advocacy and Education}

Mental health care providers need to consider community interventions, not just individual interventions, to decrease the overall community-based stigma as well as the perception of stigma. As some articles have suggested, providers need training to work against the effects of stigma as a part of their job (Crawford \& Brown, 2002). Although mental health stigma is pervasive in the United States, this research highlights how that stigma may be magnified in rural communities where confidentially concerns are high. It is not enough for social workers to simply address the concerns of any Veterans that come in their door - there are many more who are suffering and not seeking care. Working to reduce stigma throughout the community in ways that honor Veterans for their service and does not cast all Veterans as "in need" of mental health services can serve to address community-based stigma (or perhaps the perception of that stigma) for Veterans who may be have been deterred from treatment-seeking because of reputational concerns. Unlike doctors and other health and mental health providers, social workers have a strong history and training in community organization, group work, and advocacy. Given that many Veterans in rural areas make contact with their GPs as a point of entry into mental health services, it is particularly important for social workers to advocate and educate people within the health/mental health care system first, but to also address issues of stigma in the general community as well through culturally appropriate advocacy and education efforts.

\section{Research about Stigma}

Last, there needs to be more research about mental health stigma that impacts Veterans, and how the perception of others' beliefs is impacting the medical choices made by Veterans. In particular, there remains a need to better understand the specific reasons for the disparity between the need for treatment and the lack of mental healthcare service utilization among Global War on Terrorism Veterans. For example, suicide rates among this population continue to rise despite increased access to healthcare, and coordination of care seems more critical than access alone (Mills, Huber, Vince Watts, \& 
Bagian, 2011). Research has historically focused on the perceptions and beliefs of individual Veterans, but they are deeply embedded in communities that may or may not share their beliefs about seeking or receiving mental health care. More research needs to address how community stigma is impacting the rural Veteran treatment-seeking in ways that are similar to, or different, from urban Veterans.

\section{CONCLUSION}

Based on the evidence presented that stigma is a major influence in deterring treatment seeking, rural Veterans potentially face compounded challenges of experiencing both community stigma and military-related stigma. Community health care providers must understand the societal and systemic processes that influence Veterans' decision to seek mental health care and how community factors may be influencing these decisions. Rural Veterans already face many logistical barriers to care, but military and rural cultures promote values that can serve as significant psychological and social barriers to accessing treatment. Social workers have many key roles to be played in rural environments to help prevent stigmatizing attitudes and fears, to promote better organizational coordination of rural systems and social networks for Veterans, to strengthen Veterans and family members via culturally-sensitive case management and treatment services, and to conduct advocacy and education at the community level to decrease stigma at all levels in the community.

\section{References}

Adler, A. B., Bliese, P. D., McGurk, D., Hoge, C. W., \& Castro, C. A. (2009). Battlemind Debriefing and Battlemind Training as early interventions with soldiers returning from Iraq: Randomization by platoon. Journal of Consulting and Clinical Psychology, 77, 928-940

Aisbett, D.L., Boyd, C. P., Francis, K. J., Newnham, K., \& Newnham, K. (2007). Understanding barriers to mental health service utilization for adolescents in rural Australia. Rural and Remote Health, 7, 624 (online).

Bartlett, H., Travers, C., Cartwright, C., \& Smith, N. (2006). Mental health literacy in rural Queensland: results of a community survey. Australian \& New Zealand Journal of Psychiatry, 40, 783-789.

Barney, L. J., Griffiths, K. M., Jorm, A. F., \& Christensen, H. (2006). Stigma about depression and its impacts on help-seeking intentions. Australian and New Zealand Journal of Psychiatry, 40, 51-54.

Boman, E. K. O., \& Walker, G. A. (2010). Predictors of men's health care utilization. Psychology of Men \& Masculinity, 11, 113-122.

Boyd, C. P., Aisbett, D., L., Francis, K., Kelly, M., Newnham, K., \& Newnham, K. (2006). Issues in rural adolescent mental health in Australia. Rural and Remote Health, 10, 296-301. 
Boyd, C. P., Hayes, L., Sewell, J., Caldwell, K., Kemp, E., Harvie, L., et al. (2008). Commentary: Mental health problems in rural contexts: A broader perspective. Australian Psychologist, 43, 2-6.

Chapko, M. K., Borowsky, S. J., Fortney, J. C., Hedeen, A. N., Hoegle, M., Maciejewski, M. L., \& Lukas, C. V. (2002). Evaluation of the Department of Veterans Affairs Community-based outpatient clinics. Medical Care, 40, 555-560.

Chaudoir, S. R., \& Quinn, D. M. (2010). Revealing concealable stigmatized identities: The impact of disclosure motivations and positive first-disclosure experiences on fear of disclosure and well-being. Journal of Social Issues, 66, 570-584.

Corrigan, P. W., Watson, A. C., Byrne, P., \& Davis, K. E. (2005). Mental illness stigma: Problem of public health or social justice? Social Work, 50, 363-368.

Costello, T. P., Pugh, R. C., Steadman, F. B., \& Kane, R. A. (1977). Perceptions of urban versus rural hospital patients about return to their communities. Journal of the American Geriatrics Society, 25, 552-555.

Crawford, P., \& Brown, B. (2002). 'Like a friend going round': Reducing the stigma attached to mental healthcare in rural communities. Health and Social Care in the Community, 10, 229-238.

Dahlberg, K., Fosell, Y., Damström-Thakker, K., \& Runeson, B. (2007). Mental health problems and healthcare contacts in an urban and a rural area. Comparisons of two Swedish counties. Nordic Psychiatry Journal, 61, 40-46.

Ekeland, T., \& Bergem, R. (2006). The negotiation of identity among people with mental illness in rural communities. Community Mental Health Journal, 42, 225-232.

Fisher, E. S. (2003). Medical care: Is more always better? New England Journal of Medicine, 249, 1665-1667.

Fiske, H. (2003). Reflections on rural social work. Social Work Today, 3, 13-15.

Fuller, J., Edwards, J., Proctor, N., \& Moss, J. (2000). How definition of mental health problems can influence help seeking in rural and remote communities. Australian Journal of Rural Health, 8, 148-153.

Goffman, E. (1963). Stigma: Notes on the management of spoiled identity. New Jersey: Prentice Hall.

Green, R. (2003). Social work in rural areas: A personal and professional challenge. Australian Social Work, 56, 209-219.

Hinshaw, S. P. (2007). The mark of shame. Stigma of mental illness and an agenda for change. Oxford University Press: Oxford, UK.

Hoge, C. W., Castro, C. A., Messer, S. C., McGurk, D., Cotting, D. I., \& Koffman, R. L. (2004). Combat duty in Iraq and Afghanistan, mental health problems, and barriers to care. New England Journal of Medicine, 351, 13-22. 
Hoyt, D. R., Conger, R. D., Valde, J. G., \& Weihs, K., (1997). Psychological distress and help seeking in rural America. American Journal of Community Psychology, 25, 449470.

Human, J., \& Wasem, C. (1991). Rural mental health in America. American Psychologist, 46, 232-239.

Jackson, H., Judd, F., Komiti, A., Fraser, C., Murray, G., Robins, G., Pattison, P., \& Wearing, A. (2007). Mental health problems in rural contexts: What are the barrier to seeking help from professional providers? Australian Psychologist, 42(2), 147-160.

Jagdeo, A., Cox, B. J., Stein, M. B., \& Sareen, J. (2009). Negative attitudes toward help seeking for mental illness in two population-based surveys from the United States and Canada. The Canadian Journal of Psychiatry, 54, 757-766.

Jerrell, J. M., \& Knight, M. A. (1985). Social work practice in rural mental health systems. Social Work, 30, 331-337.

Jones, E., Farina, A., Hastorf, A., Markus, H., Miller, D., \& Scott, R. (1984). Social stigma: The psychology of marked relationships. New York: Freeman.

Judd, F., Jackson, H., Komiti, A., Murray, G., \& Fraser, C. (2007). Service utilisation by rural residents with mental health problems. Australasian Psychiatry, 15(3), 185-190.

Judd, F., Jackson, H., Komiti, A., Murray, G., Fraser, C., Grieve, A., \& Gomez, R., (2006). Help-seeking by rural residents for mental health problems: The importance of agrarian values. Australian and New Zealand Journal of Psychiatry, 40, 769-776.

Kirchner, J. E., Cody, M., Thrush, C. R., Sullivan, G., \& Rapp, C. G. (2004). Identifying factors critical to implementation of integrated mental health services in rural VA community-base outpatient-based clinics. Journal of Behavioral Health Services and Research, 31, 13-25.

Kline, A., Falca-Dodson, M., Sussner, B., Ciccone, D. S., Chandler, H., Callahan, L, \& Losonczy, M. (2010). Effects of repeated deployment to Iraq and Afghanistan on the health of New Jersey Army National Guard troops: Implications for military readiness. American Journal of Public Health, 100, 276-283.

Landsman, M. J. (2002). Rural child welfare practice from an organization-inenvironment perspective. Child Welfare, 81, 249-261.

Levin, A. (2001). Violence and mental illness: Media keep myths alive. Psychiatric News, 36, 10.

Link, B. G., \& Phelan, J. C. (2001). Conceptualizing stigma. Annual Review of Sociology, 27, 363-385.

Lorber, W., \& Garcia, H. S. (2010). Not supposed to feel this: Traditional masculinity in psychotherapy with male Veterans returning from Afghanistan and Iraq. Psychotherapy Theory, Research, Practice, and Training, 47, 296-305. 
Mills, P. D., Huber, S. J., Vince Watts, B., \& Bagian, J. P. (2011). Systemic vulnerabilities to suicide among Veterans from the Iraq and Afghanistan conflicts: Review of case reports from a national Veterans Affairs database. Suicide and Life Threatening Behavior, 41, 21-32.

Mohamed, S., Neale, M. S., \& Rosenheck, R. (2009). Veterans Affairs intensive case management for older Veterans. American Journal of Geriatric Psychiatry, 17, 671681.

Molinari, V., Boeve, W., Kunik, M., Snow-Turek, A. (1999). Rural vs. urban geropsychiatric patients: Differences on demographic and psychiatric measures. Clinical Gerontologist, 20, 45-55.

National Rural Health Association. (2004). Rural Veterans: A Special Concern for Rural Health Advocates. Retrieved from www.NRHArural.org

Parr, H., \& Philo, C. (2003). Rural mental health and social geographies of caring. Social and Cultural Geography, 4, 47-488.

Parr, H., Philo, C., \& Burns, N. (2004). Social geographies of rural mental health: Experiencing inclusion and exclusion. Transactions of the Institute of British Geographers, 29, 401-419.

Pietrzak, R. H., Johnson, D. C., Goldstein, M. B., Malley, J. C., \& Southwick, S. M. (2009). Perceived stigma, barriers to care, and mental health service utilization in Veterans of Operations Enduring Freedom and Iraqi Freedom: The importance of unit support and beliefs about mental healthcare. Psychiatric Services, 60, 11181122 .

Quine, S., Bernard., D., Booth, M., Kant, M., Usherwood, T., Alperstein, G., et al. (2003). Heath and access issues among Australian adolescents: A rural - urban comparison. Rural and Remote Health, 3, 245 (online).

Reivich, K. J., Seligman, M. E. P., \& McBride, S. (2011). Master resilience training in the U.S. Army. American Psychologist, 66, 25-34.

Riebschleger, J. (2007). Social workers' suggestions for effective rural practice. Families in Society, 88, 203-213.

Savitsky, L., Illingworth, M., \& DuLaney, M. (2009). Civilian social work: Serving the military and Veteran populations. Social Work, 54, 327-339.

Sayer, N. A., Friedemann-Sanchez, G., Spoont, M., Murdoch, M., Parker, L., Chiros, C., \& Rosenheck, R. (2009). A qualitative study of determinants of PTSD treatment initiation in Veterans. Psychiatry, 72, 238-255.

Shipard, J. C., \& Beck, J. G. (2005). The role of thought suppression in posttraumatic stress disorder. Behavior Therapy, 26, 277-287.

Smart, L., \& Wegner, D. M. (1999). Covering up what can't be seen: Concealable stigma and mental control. Journal of Personality and Social Psychology, 77, 474-486. 
Sayer, N. A., Friedemann-Sanchez, G., Spoont, M., et al. (2009). A qualitative study of determinants of PTSD treatment initiation in Veterans. Psychiatry, 72, 238-255.

Stecker, T., Fortney, J. C., Hamilton, F., \& Ajzen, I. (2007). An assessment of beliefs about mental health care among Veterans who served in Iraq. Psychiatric Services, $58,1358-1361$.

Wahl, O. F. (1999). Mental health consumers' experience of stigma. Schizophrenia Bulletin, 25, 467-478.

Wallace, A., Weeks, W., Wang, S., Lee, A., \& Kazis, L. (2006). Rural and urban disparities in health-related quality of life among Veterans with psychiatric disorders. Psychiatric Services, 57, 851-856.

Warner, C. H., Appenzeller, G. N., Mullen, K., Warner, C. M., \& Grieger, T. (2008). Soldier attitudes toward mental health screening and seeking care upon return from combat. Military Medicine, 173, 563-569.

Weeks, W. B., Kazis, L. E., Shen, Y., Cong, Z., Ren, X. S., Miller, D., Lee, A., \& Perlin, J. B. (2004). Differences in health-related quality of life in rural and urban Veterans. American Journal of Public Health, 94, 1762-1767.

Weeks, W. B., Mahar, P. J., Wright, S. M. (2005). Utilization of VA and medicare services by medicare-eligible Veterans: The impact of additional access points in a rural setting. Journal of Healthcare Management, 50(2), 95-106.

West, A., \& Weeks, W. B. (2006). Physical and mental health and access to care among nonmetropolitan Veterans Health Administration patients younger than 65 years. Journal of Rural Health, 22, 9-16.

Wright, K. M., Cabrera, O. A., Bliese, P. D., Adler, A. B., Hoge, C. W., \& Castro, C. A. (2009). Stigma and barriers to care in soldiers postcombat. Psychological Services, 6, 108-116.

Wrigley, S., Jackson, H., Judd, F., \& Komiti, A. (2005). Role of stigma and attitudes toward help-seeking from a general practitioner for mental health problems in a rural town. Australian and New Zealand Journal of Psychiatry, 39, 514-521.

\section{Author's note:}

Address correspondence to: Professor Rebecca L. Stotzer, University of Hawai' i, Mānoa 1800 East West Rd., Henke Hall, Honolulu, HI 96822. Email: rstotzer@hawaii.edu 